\title{
Save the Brain First: CTA and Mechanical Thrombectomy in Patients at Risk for Contrast-Induced Nephropathy
}

S troke is one of the most common diseases affecting 800,000 Americans each year. It ranks fourth among all-cause mortality in the United States. ${ }^{1}$ When patients present with stroke-like symptoms, the current American Heart Association/American Stroke Association (AHA/ASA) guidelines recommend an immediate non-contrast CT of the head. If large-vessel occlusion is suspected, CTA or MRA should be performed to assess the vascular anatomy and plan for endovascular therapy. If the patient is a candidate for mechanical thrombectomy, cerebral angiography is performed. $^{2}$ Both CTA and mechanical thrombectomy will subject patients to intravenous contrast.

The concern for exposing patients to a large amount of intravenous contrast is the development of contrast-induced nephropathy (CIN). It is defined as an increase in the plasma creatinine level of $0.5 \mathrm{mg} / \mathrm{dL}$ or $>25 \%$ increase from the baseline within 25 days of contrast exposure without any other attributable cause. ${ }^{3}$ Multiple risk factors can predispose patients to CIN. Tsai et $\mathrm{al}^{4}$ have shown that severe chronic kidney disease (CKD), defined as an estimated glomerular filtration rate (eGFR) of $<30 \mathrm{~mL} / \mathrm{min} /$ $1.72 \mathrm{~m}^{2}$, was the most significant risk factor for the development of CIN. Most important, acute kidney injury (AKI) in patients with stroke has been associated with an increased risk of in-hospital mortality. ${ }^{5}$ Therefore, CIN is a relevant clinical entity and needs to be managed appropriately.

When the initial head CT is negative for bleeding, the question arises as to when and whether the patient should undergo CTA and subsequent revascularization. The current AHA/ASA guidelines recommend proceeding with CTA in patients without a history of renal disease. ${ }^{2}$ However, for patients with a history of CKD, there is no current consensus guideline. Therefore, we believe the article by Myung et $\mathrm{al}^{6}$ has shed valuable light on the risk-stratification and clinical decision-making for this patient population.

The authors conducted a retrospective observational study examining the relationship between CIN and baseline renal function in a large cohort involving 601 patients undergoing CTA and cerebral angiography. The authors demonstrated that patients with severe CKD (eGFR of $<30 \mathrm{~mL} / \mathrm{min} / 1.73 \mathrm{~m}^{2}$ ) were at a higher risk of CIN $(P<.001)$. The $\mathrm{CIN}$ incidence rate was $12.5 \%$ in all patients with $\mathrm{CKD}$ and $46.6 \%$ in patients with severe CKD.
Furthermore, the cutoff eGFR value for an increased CIN risk was found to be $43 \mathrm{~mL} / \mathrm{min} / 1.73 \mathrm{~m}^{2}$. Most important, there was no reported mortality in the patients with CIN. Only 5 patients underwent dialysis, and all patients fully recovered renal function within 4 days. Due to its retrospective observational nature, in this study, a causal relationship could not be established between intravenous contrast and CIN. Furthermore, the absolute CIN incidence rate in patients with $\mathrm{CKD}$ was low. Patients with severe $\mathrm{CKD}$ were at an increased risk of CIN. However, the clinical consequence is not necessarily significant because all patients recovered successfully after a short course of dialysis and no in-hospital mortality was reported. The authors concluded that neurological interventions should take precedence over concerns of renal injury. However, the understanding of CIN risk factors may help guide individualized renal protective therapy.

Similar to the authors' conclusion, it is absolutely reasonable to proceed with endovascular therapy regardless of the patient's baseline renal function. It is important to proceed with neurovascular interventions before the creatinine level is drawn to avoid a prolonged time to intervention.

First, there is a growing body of evidence showing that intravenous contrast may not cause CIN, particularly in patients with unremarkable baseline renal function. A 2013 systematic review and metaanalysis showed no difference in the rate of AKI between patients with and without contrast exposure. ${ }^{7}$ In 2017 , a systematic review of 14 studies revealed that CTA and CT perfusion scans were not significantly associated with an increased risk of developing AKI. ${ }^{8}$ Furthermore, Lima et $\mathrm{al}^{9}$ have shown that there was no difference in the AKI rate between patients undergoing CTA and thrombectomy and those without any contrast exposure. Other studies have found similar results. ${ }^{10,11}$ Of course, the major critique is that high-risk patients might be scanned much less frequently than the generally healthy patient population owing to the concern for CIN. Therefore, the CIN incidence in the contrast-exposure group may have been erroneously low. On the other hand, stroke may disrupt neurohormonal pathways and may contribute to the development of AKI. ${ }^{12}$

Second, although CIN has been reported to range as high as $20 \%-30 \%$ in the high-risk renal population, the true incidence rate may be much lower. A 2013 study showed that $9 \%-11 \%$ of the at- 
risk patients developed postcontrast AKI. ${ }^{13}$ A 2017 meta-analysis showed that $2.3 \%$ of the patients with CKD undergoing CTA were affected by AKI. ${ }^{7}$ Similarly, other studies have found that the absolute CIN rate was low in at-risk patients. ${ }^{14}$ The often-quoted high CIN incidence rate was partially due to the use of older hyperosmolar contrast agents, which have been associated with an increased risk of CIN. ${ }^{15}$ Furthermore, the incidence of CIN in patients undergoing neurovascular therapy is also low. In a study including 185 high-risk patients undergoing cerebral angiography and mechanical thrombectomy, only 1 patient developed CIN. ${ }^{16}$ Similarly, Loh et $\mathrm{al}^{17}$ found that 3 of 99 patients undergoing endovascular therapy had AKI. In a more recent study, 12 of 93 patients with CKD developed AKI after CTA and thrombectomy, though CKD was not an independent risk factor for AKI in this study. ${ }^{18}$

Last, although CIN has been associated with an increased in-hospital mortality, the true clinical significance of CIN may also be overestimated. It is plausible that CIN is a surrogate marker of rather than a causative factor for worse clinical outcome. For example, the degree of renal impairment is typically minor. $\mathrm{McDonald}$ et $\mathrm{al}^{7}$ have shown that the rate of dialysis was $0.3 \%$ in patients developing CIN. Furthermore, effort to reduce renal impairment does not improve the overall mortality rate. Coca et $\mathrm{al}^{19}$ have shown that reducing the AKI rate by $>50 \%$ did not reduce the risk of long-term mortality.

Currently, there is no prospective, randomized controlled trial comparing the risk of AKI in patients with CKD with and without contrast exposure. Therefore, no true causal relationship can be established. However, based on existing literature, there is evidence indicating that intravascular contrast does not cause CIN. Furthermore, the absolute rate of CIN remains low, and the clinical significance is unclear. It is more likely that CIN is a surrogate marker reflecting critical illness and multimorbidity rather than a factor determining patient outcome. In that case, assessing a patient's renal function before CTA and mechanical thrombectomy may have no clinical benefit from a renal-protective perspective, even for patients with CKD. Furthermore, each minute wasted on obtaining laboratory data may cost patients 1.9 million neurons in the stroke population. ${ }^{20}$ Therefore, it is prudent to proceed with CTA and mechanical thrombectomy on the basis of neurologic status regardless of the patient's renal function. In the future, prospective, randomized controlled trials for patients with severe CKD may be considered, but ethically, it will be difficult not to treat patients if they are otherwise candidates for mechanical thrombectomy. Time is indeed brain, and brain should be saved first.

\section{REFERENCES}

1. Koton S, Schneider AL, Rosamond WD, et al. Stroke incidence and mortality trends in US communities, 1987 to 2011. JAMA 2014;312:259-68 CrossRef Medline

2. Powers WJ, Rabinstein AA, Ackerson T, et al; American Heart Association Stroke Council. 2018 Guidelines for the Early Management of Patients with Acute Ischemic Stroke: A Guideline for Healthcare Professionals From the American Heart Association/American Stroke Association. Stroke 2018;49:e46-110 CrossRef Medline

3. Nijssen EC, Rennenberg RJ, Nelemans PJ, et al. Prophylactic hydration to protect renal function from intravascular iodinated contrast material in patients at high risk of contrast-induced nephropathy (AMACING): a prospective, randomised, phase 3, controlled, openlabel, non-inferiority trial. Lancet 2017;389:1312-22 CrossRef Medline
4. Tsai TT, Patel UD, Chang TI, et al. Contemporary incidence, predictors, and outcomes of acute kidney injury in patients undergoing percutaneous coronary interventions: insights from the NCDR cath-PCI registry. JACC Cardiovasc Interv 2014;7:1-9 CrossRef Medline

5. Gadalean F, Simu M, Parv F, et al. The impact of acute kidney injury on in-hospital mortality in acute ischemic stroke patients undergoing intravenous thrombolysis. PLoS One 2017;12:e185589 CrossRef Medline

6. Myung JW, Kim JH, Cho JH, et al. Contrast-induced acute kidney injury in radiologic management of acute ischemic stroke in the emergency setting. AJNR 2020;41:632-36 CrossRef Medline

7. McDonald JS, McDonald RJ, Comin J, et al. Frequency of acute kidney injury following intravenous contrast medium administration: a systematic review and meta-analysis. Radiology 2013;267:119-28 CrossRef Medline

8. Brinjikji W, Demchuk AM, Murad MH, et al. Neurons over nephrons; systematic review and meta-analysis of contrast-induced nephropathy in patients with acute stroke. Stroke 2017;48:1862-68 CrossRef Medline

9. Lima FO, Lev MH, Levy RA, et al. Functional contrast-enhanced CT for evaluation of acute ischemic stroke does not increase the risk of contrast-induced nephropathy. AJNR Am J Neuroradiol 2010;31:81721 CrossRef Medline

10. Jia ZY, Wang SX, Zhao LB, et al. Risk of acute kidney injury with consecutive, multidose use of iodinated contrast in patients with acute ischemic stroke. AJNR Am J Neuroradiol 2019;40:652-54 CrossRef Medline

11. Langner S, Stumpe S, Kirsch M, et al. No increased risk for contrastinduced nephropathy after multiple CT perfusion studies of the brain with a nonionic, dimeric, iso-osmolal contrast medium. AJNR Am J Neuroradiol 2008;29:1525-29 CrossRef Medline

12. Zhao Q, Yan T, Chopp M, et al. Brain-kidney interaction: renal dysfunction following ischemic stroke. J Cereb Blood Flow Metab 2020;40:246-62 CrossRef Medline

13. McDonald RJ, McDonald JS, Bida JP, et al. Intravenous contrast material-induced nephropathy: causal or coincident phenomenon? Radiology 2013;267:106-18 CrossRef Medline

14. Hopyan JJ, Gladstone DJ, Mallia G, et al. Renal safety of CT angiography and perfusion imaging in the emergency evaluation of acute stroke. AJNR Am J Neuroradiol 2008;29:1826-30 CrossRef Medline

15. Lautin EM, Freeman NJ, Schoenfeld AH, et al. Radiocontrast-associated renal dysfunction: a comparison of lower-osmolality and conventional high-osmolality contrast media. Am J Roentgenol 1991;157:59-65 Medline

16. Kim J, Male S, Jagadeesan BD, et al. Safety of cerebral angiography and neuroendovascular therapy in patients with chronic kidney disease. Neuroradiology 2018;60:529-33 CrossRef Medline

17. Loh Y, McArthur DL, Vespa P, et al. The risk of acute radiocontrast-mediated kidney injury following endovascular therapy for acute ischemic stroke is low. AJNR Am J Neuroradiol 2010;31:158487 CrossRef Medline

18. Weber $\mathrm{R}$, van Hal $\mathrm{R}$, Stracke $\mathrm{P}$, et al. Incidence of acute kidney injury after computed tomography angiography \pm computed tomography perfusion followed by thrombectomy in patients with stroke using a postprocedural hydration protocol. J Am Heart Assoc 2020;9:e014418 CrossRef Medline

19. Coca SG, Zabetian A, Ferket BS, et al. Evaluation of short-term changes in serum creatinine level as a meaningful end point in randomized clinical trials. J Am Soc Nephrol 2016;27:2529-42 CrossRef Medline

20. Saver JL. Time is brain: quantified. Stroke 2006;37:263-66 CrossRef

$$
\begin{array}{r}
\text { (1) X. Li } \\
\text { Section of Interventional Radiology, Imaging Institute } \\
\text { Cleveland Clinic Foundation }
\end{array}
$$

Cleveland, Ohio

http://dx.doi.org/10.3174/ajnr.A6515 